\title{
COMMUTATIVE RINGS WITH IDENTITY HAVE RING TOPOLOGIES
}

\author{
BY M. HOCHSTER AND J. O. KILTINEN ${ }^{1}$
}

Communicated by Leonard Gillman, October 16, 1969

Throughout, let $R$ denote a commutative ring with identity. By a proper topology we mean a separated nondiscrete ring topology.

THEOREM. Every infinite $R$ has a proper topology.

The following five propositions outline the proof. Details will appear in [2].

Proposition 1. Each infinite $R$ satisfies at least one of the following conditions.

(a) $R$ admits a proper ideal topology (i.e. one having a neighborhood basis at 0 consisting of ideals).

(b) $R$ contains infinitely many nilpotents.

(c) There is an element $r \in R$ such that $R / \mathrm{Ann}_{R} r$ is an infinite field.

The proof depends on the characterizations of rings with proper ideal topologies in [1]. To prove the theorem we now need only consider rings satisfying (b) or (c).

Proposition 2. Let $I$ be an ideal of $R$ having a proper R-algebra topology $J$ ( $R$ discrete). There is a unique proper topology on $R$ such that $I$ (with topology $\mathrm{J}$ ) is an open subspace.

Proposition 3. Let $\phi: R / \operatorname{Ann}_{R} r \rightarrow(r)$ be the obvious $R$-module isomorphism. For each proper topology on $R / \mathrm{Ann}_{R} r, \phi$ induces a proper $R$-algebra topology on $(r)$. Hence, if $R / \mathrm{Ann}_{R} r$ has a proper topology, so does $R$.

It is known that all infinite fields have proper topologies [3, Theorem 5.2, p. 159]. With this result and Proposition 3 we have

Corollary. If $R$ satisfies (c), $R$ has a proper topology.

Proposition 4. Every infinite abelian group $I$ has a separated nondiscrete group topology such that every endomorphism of $I$ is continuous.

AMS Subject Classifications. Primary 1698, 1350; Secondary 1270, 1320, 2210.

Key Words and Phrases. Topological ring, commutative ring with identity, existence of ring topologies, ideal topology.

1 Both authors were supported in part by NSF Grant GP-8496. 
(The proof is an easy consequence of Pontrjagin duality.)

Proposition 5. Let $R$ satisfy (b). Then there is an infinite ideal $I$ of $R$ such that $I^{2}=(0)$. If $J$ is an additive group topology for $I$ as in Proposition 4 , then $J$ is a proper $R$-algebra topology for $I$. Hence, by Proposition 2, $R$ has a proper topology.

\section{REFERENCES}

1. M. Hochster, Rings with nondiscrete ideal topologies, Proc. Amer. Math. Soc. 21 (1969), 357-362.

2. - Existence of topologies for commutative rings with identity (to appear). 3. J. O. Kiltinen, Inductive ring topologies, Trans. Amer. Math. Soc. 134 (1968), 149-169. MR 37 \#054.

University of Minnesota, Minneapolis, Minnesota 55455 